\title{
Short Length Trellis-Based Codes for Gaussian Multiple-Access Channels
}

\author{
Ayça Özçelikkale and Tolga M. Duman
}

\begin{abstract}
We focus on trellis-based joint code design for twouser Gaussian multiple-access channel (MAC) in the short block length regime. We propose a design methodology, provide specific code designs and report numerical performance results. We compare the performance of the jointly designed codes with the performance of the codes designed for point-to-point (P2P) channels including optimum (in terms of minimum distance) convolutional codes. Our results show that the proposed codes achieve superior performance compared to these alternatives especially in the high signal-to-noise (SNR) regime in equal power scenarios.
\end{abstract}

Index Terms-Code design, convolutional codes, multiple-access channel.

\section{INTRODUCTION}

A NALYSIS of fundamental limits of information transmission, as well as design of channel coding solutions typically focus on communication scenarios where codes with long block lengths are used. On the other hand, practical applications with hardware complexity, battery life or delay constraints may require information transmission with short blocks. For instance, wireless sensor networks built for real-time control or surveillance have tight latency requirements, suggesting usage of short block lengths. Motivated by these observations, we investigate practical channel coding solutions in the short block length regime. We consider Gaussian multiple-access channel, and focus on terminated convolutional codes.

Existing research on code design with short block lengths demonstrates promising results for point-to-point channel with convolutional codes in terms of the performance gap with Shannon's sphere packing bound [1], which is a fundamental performance evaluation tool [2], [3]. Convolutional codes also provide an attractive alternative from a design point of view as well as from an efficient decoding perspective for MAC channels in the short block length framework. It is possible to find weight distribution functions for individual instances

Manuscript received April 10, 2014; revised May 17, 2014; accepted May 17, 2014. Date of current version June 05,2014 . This publication was made possible by NPRP Grant 4-1293-2-513 from Qatar National Research Fund (a member of Qatar Foundation). The statements made herein are solely the responsibility of the authors. This work was performed when A. Özçelikkale was with Bilkent University, Turkey. The associate editor coordinating the review of this manuscript and approving it for publication was Prof. Chandra Ramabhadra Murthy.

A. Özçelikkale is with the Department of Signals and Systems, Chalmers University of Technology, SE-41296 Gothenburg, Sweden (e-mail: ayca.ozcelikkale@chalmers.se).

T. M. Duman is with the Department of Electrical Engineering, Bilkent University, TR-06800 Ankara, Turkey, on leave from Arizona State University, Tempe, AZ 85004 USA (e-mail: duman@ee.bilkent.edu.tr).

Color versions of one or more of the figures in this paper are available online at http://ieeexplore.ieee.org.

Digital Object Identifier 10.1109/LSP.2014.2328330 of convolutional codes, making it feasible to compute performance bounds in various scenarios, see e.g. [4], [5]. This is in contrast to low density parity check (LDPC) codes, where the traditional methods for code performance evaluation, such as EXIT charts, focus on the asymptotic regime (in the block length). Moreover, it is possible to develop optimal (in the maximum-a-posteriori (MAP) or maximum likelihood (ML) sense) decoding algorithms if trellis-based codes are employed at both users. Another point that makes convolutional codes attractive in MAC scenario is the similarities between the space-time coding (STC) scenario and the MAC scenario, and the good performance of trellis-based codes in STC scenarios [6]-[10].

Motivated by these observations, we focus on convolutional (trellis-based) coding framework for short block length codes over Gaussian multiple access channels. We propose a design methodology, provide specific code designs and report numerical performance results. We compare performance of the designed codes with the performance of the optimum (in terms of minimum distance) convolutional codes designed for P2P channels, and available short length LDPC codes. We illustrate how jointly designed and decoded convolutional codes achieve superior performance compared to the strategy of using codes designed for P2P channels. Our results indicate that it is particularly important to jointly design codes when the users have equal power and the regime of operation is high SNRs.

The paper is organized as follows: in Section II, the system model is described. Performance analysis and the proposed joint convolutional code design approach are described in Section III. Numerical performance results for the designed codes are reported in Section IV. The paper is concluded in Section V.

\section{SYSTEM MODEL}

We consider a multiple-access channel with two users. The users separately encode their messages into length- $n$ codewords $\mathbf{c}^{1}$ and $\mathbf{c}^{2}$, where $\mathbf{c}^{i}=\left[\begin{array}{llll}c_{1}^{i} & c_{2}^{i} & \ldots & c_{n}^{i}\end{array}\right]$, with $c_{t}^{i}$ representing the output symbol transmitted from user $i, i=1,2$ at time $t$. The received signal $y_{t}$ at time $t$ can be expressed as follows

$$
y_{t}=\alpha^{1} c_{t}^{1}+\alpha^{2} c_{t}^{2}+w_{t}
$$

where $\alpha^{i}$ 's denote the real and fixed channel gains, and $w_{t}$ represents the i.i.d. zero mean Gaussian noise with variance $N_{0} / 2$. Let $\mathbf{c}$ be the codeword matrix of size $2 \times n$ that represents the codewords for both users together as follows

$$
\mathbf{c}=\left[\begin{array}{llll}
c_{1}^{1} & c_{2}^{1} & \ldots & c_{n}^{1} \\
c_{1}^{2} & c_{2}^{2} & \ldots & c_{n}^{2}
\end{array}\right]=\left[\begin{array}{l}
\mathbf{c}^{1} \\
\mathbf{c}^{2}
\end{array}\right]
$$


The model for transmission of one frame can be expressed more compactly as follows

$$
\mathbf{y}=\boldsymbol{\alpha} \mathbf{c}+\boldsymbol{w},
$$

where $\boldsymbol{\alpha}=\left[\alpha^{1} \alpha^{2}\right], \mathbf{y}=\left[y_{1} \ldots y_{n}\right]$ and $\boldsymbol{w}=\left[w_{1} \ldots w_{n}\right]$.

\section{Performance AnAlysis}

\section{A. Pairwise Error Probability}

Upon receiving $\mathbf{y}$, the receiver produces the estimates of the codewords $\hat{\mathbf{c}}^{1}$ and $\hat{\mathbf{c}}^{2}$. It is desired to send both codewords reliably so that the error event is defined as

$$
\mathcal{E} \doteq\left\{\hat{\mathbf{c}}^{1} \neq \mathbf{c}^{1}\right\} \cup\left\{\hat{\mathbf{c}}^{2} \neq \mathbf{c}^{2}\right\} .
$$

The error event can be also expressed in terms of the two user codeword matrix $\mathbf{c}$ as $\mathcal{E}=\{\hat{\mathbf{c}} \neq \mathbf{c}\}$.

The optimal decoder decides according to the minimum Euclidean distance criterion. The pairwise error probability that the received signal is closer to another codeword pair $\hat{\mathbf{c}}$ instead of c when $\mathbf{c}$ was transmitted can be expressed as follows [4], [5]

$$
P_{\mathcal{E}}(\mathbf{c}, \hat{\mathbf{c}})=Q\left(\sqrt{\frac{d^{2}(\mathbf{c}, \hat{\mathbf{c}})}{2 N_{0}}}\right),
$$

where $Q(x)=\frac{1}{\sqrt{2 \pi}} \int_{x}^{\infty} \exp \left(-\frac{1}{2} t^{2}\right) d t$, and $d^{2}(\mathbf{c}, \hat{\mathbf{c}})$ is the squared Euclidean distance between $\hat{\mathbf{c}}$ and $\mathbf{c}$. It can be expressed as follows

$$
d^{2}(\mathbf{c}, \hat{\mathbf{c}})=\boldsymbol{\alpha} \mathbf{D}_{\mathbf{c}, \hat{\mathbf{c}}} \boldsymbol{\alpha}^{\dagger}
$$

where

$$
\mathbf{D}_{\mathbf{c}, \hat{\mathbf{c}}} \doteq(\mathbf{c}-\hat{\mathbf{c}})(\mathbf{c}-\hat{\mathbf{c}})^{\dagger},
$$

is the two user codeword difference correlation matrix. Here $\dagger$ denotes the transpose.

\section{B. Union Bound}

Using the union bound, the frame error probability can be upper-bounded as follows

$$
P_{f} \leq \frac{1}{|\mathcal{C}|} \sum_{\mathbf{c}} \sum_{\mathbf{c} \neq \hat{\mathbf{c}}} P_{\mathcal{E}}(\mathbf{c}, \hat{\mathbf{c}}),
$$

where $\mathcal{C}$ denotes the set of codeword pairs $\mathbf{c}$, and |.| denotes the cardinality of the set.

\section{Product Weight Enumeration for Convolutional Codes}

Although the above analysis holds for any given code, in general it is not possible to efficiently calculate the bound in (8), due to the complexity of enumeration of the multiplicities of the matrices $\mathbf{D}_{\mathbf{c} . \hat{\mathbf{c}}}$ for all possible correct-erroneous codeword pairs. An exception is the case of convolutional (or trellis-based) codes, for which this calculation can be done systematically. In this section, we discuss this point, and give details on how to do this efficiently.

Let $s_{i}$ and $M_{i}$ denote the state of the trellis and the state transition matrix for user $i$. Using $M_{i}$ one can calculate the individual weight enumerators for user $i$. Instead, we consider the state transitions for the two users jointly and define a two-user joint trellis which represents the state transitions for both users. The states in this two-user joint trellis are in the form $\left(s_{1}, s_{2}\right)$, where $s_{i}$ denotes the state of the path for $i$ th user, i.e. state for the path for the codeword $\mathbf{c}^{i}$. There are $n_{s_{1}} \times n_{s_{2}}$ such states, where $n_{s_{i}}$ is the number of states for the $i$ th user's code. We represent the associated state transition matrix with $M_{12}$. Using $M_{12}$, weight enumeration for the joint two user code can be found.

In order to be able to evaluate the performance, two user codeword difference correlation matrix given in (7) for all possible codeword pairs $(\mathbf{c}, \hat{\mathbf{c}})$ have to be tracked, i.e. multiplicities of all the possible $\mathbf{D}_{\mathbf{c}, \hat{\mathbf{c}}}$ 's over the joint code of the two users should be found. For this purpose, a product state trellis is defined, where the states are in the form $(\mathbf{s}, \hat{\mathbf{s}})$. Here $\mathbf{s}=\left(\mathbf{s}_{1}, \mathbf{s}_{2}\right)$ and $\hat{\mathbf{s}}=\left(\hat{\mathbf{s}}_{1}, \hat{\mathbf{s}}_{2}\right)$ denote the state of the path for $\mathbf{c}$ and $\hat{\mathbf{c}}$, respectively. There are $\bar{n}_{s}=\left(n_{s_{1}} \times n_{s_{2}}\right)^{2}$ such states. The associated state transition matrix is labeled as $\bar{M}_{12}$. The entries of $\bar{M}_{12}$ are either zero corresponding to the case where the transition is not allowed, or in the form

$$
\left[\bar{M}_{12}\right]_{k, l}=D_{11}^{q_{11}^{k, l}} \times D_{12}^{q_{12}^{k, l}} \times D_{22}^{q_{11}^{k, l}}, \quad k, l=1, \ldots \bar{n}_{s}
$$

where $[M]_{k, l}$ is the $k$ th row $l$ th column entry of the matrix $M$. Here $D_{i j}, i, j=1,2$ are dummy variables, and the exponent $q_{i j}^{k, l}$ gives the contribution of the transition from state $k$ to state $l$ to the $i$ th row $j$ th column entry of the codeword different correlation matrix $\mathbf{D}_{\mathbf{c}, \hat{\mathbf{c}}}$. We note that since we have $\left[\mathbf{D}_{\mathbf{c}, \hat{\mathbf{c}}}\right]_{1,2}=\left[\mathbf{D}_{\mathbf{c}, \hat{\mathbf{c}}}\right]_{2,1}^{\dagger}$, we only need to keep track of one of these parameters.

In order to tighten the bounds, expurgation technique is adopted and only simple error events are considered [4], [11]. For simple error events, the codeword matrices $\mathbf{c}$ and $\hat{\mathbf{c}}$ only differ in one segment of the path. To keep track of simple error events, we use an extended state diagram where an extra state is introduced in $\bar{M}_{12}$. Let us denote this state as $\mathbf{s}_{\mathbf{e}}$. Transition to $\mathrm{s}_{\mathrm{e}}$ is done only from the states where an error has occurred, i.e. where the states corresponding to $\mathbf{c}$ and $\hat{\mathbf{c}}$ differ. From such an error state, transition is done to $\mathbf{s}_{\mathbf{e}}$ when the error event ends, i.e. the simple error event terminates. If the path enters this state, the only possible transition is to stay in this state.

For an $L$ stage trellis, by calculating the $L$ th power of $\bar{M}_{12}$, and accounting for trellis termination, we find the complete list of possible $\mathbf{D}_{\mathbf{c}, \hat{\mathbf{c}}}$ and their multiplicities. These quantities can then be used to compute the union bound on the error rate of the code pair.

\section{Code Search Approach}

We use the frame error rate bound in (8) evaluated using the product weight enumeration method presented in Section III-C as the performance criteria in our code search. This method jointly determines the codes for both users.

In Section III-C, it is assumed that enumeration of the weights is done over the duration of the entire frame. The computational cost of such an operation is high [5], making its direct usage for the purpose of code search a poor choice. Hence, we consider the following simplification: a shorter frame length than the intended design length is used for weight enumeration. This modification is motivated by the general nature of convolutional codes, and the related observation that for decoding of convolutional codes, it is possible to obtain satisfactory performance 
with a traceback length of four or five times the constraint length $m[12$, Ch.4]. Hence actual block length of the codes may not be crucial in the design of the convolutional code. Our numerical experiments suggest that for a sufficiently long length, (again five times $m$ is a good rule of thumb), the general performance rankings of the candidate codes are preserved.

Another simplification adopted for computational efficiency is related to the magnitude of the entries of $\mathbf{D}_{\mathbf{c}, \hat{\mathbf{c}}}$. We drop out the terms with magnitude greater than a threshold as they do not have an appreciable effect on the error bounds, similar to the approaches in [4], [5] presented in the space-time coding framework. This threshold is chosen according to the constraint length of the codes for which the search is being performed and the chosen modulation.

\section{E. Alternative Code Design Approaches}

We now compare our code design approach with some alternative approaches. Although here we focus on convolutional codes, another option would be to use LDPC codes. Indeed, excellent performance results for LDPC codes are reported for the long block length regime for multi-user channels (e.g. Gaussian MAC, broadcast channel) [13], [14]. Nevertheless, design and use of LDPC codes as short block length codes is problematic. This stems from both difficulties that exist in the short length scheme for LDPC codes even for P2P channels, and also from difficulties specific to the MAC channel. Code design with LDPC codes in multi-user scenarios typically utilize tools designed specifically for the long block length scheme [13], [14]. Although there is a small number of works that focus on the design of LDPC codes with short block lengths for P2P channels [15], [16], it is not straightforward to extend these approaches for the MAC channel.

This lack of joint design tools is not a matter that can be easily dismissed. To demonstrate this point, we now give an example which illustrates using strong codes that are designed for P2P channels is, in general, not the optimal strategy even when ML decoding is possible. In our example, we use the $(24,12)$ extended binary Golay code. The minimum distance of this code is 8 , hence it is very attractive in a P2P setting for $n=24$. Moreover, due to the short length of the code, for error performance comparison purposes, it is possible to perform joint $\mathrm{ML}$ decoding. To use this code in the MAC scenario, we consider the strategy where one of the users encodes his/her message using a Golay code, and the other user employs an interleaved version. ${ }^{1}$ The error performance of Golay code pair is observed to be very poor compared to the designed codes: the frame error rate (FER) for the strategy of using Golay code is found to be larger than 0.1 at $8 \mathrm{~dB}$ for all of the 10 arbitrarily chosen interleavers. On the other hand, with a code found by the proposed method, $\mathcal{C}_{1}=(6,3) /(5,5)$, the FER value is found to be as low as 0.0035 . (In order to have a convolutional code with the exact rate of $1 / 2,14$ bits including the terminating zeros are encoded, and the output codeword is punctured at the $\{1,11,16,27\}$ th bits.)

Another drawback that makes the usage of LDPC codes difficult is the lack of (known) efficient (near-optimal) decoding

\footnotetext{
${ }^{1}$ We note that the two codes at the two users cannot be identical, otherwise it would be impossible to differentiate the two separate messages at the destination, hence we interleave one of them for use over MAC.
}

algorithm for short length LDPC codes on MAC channel. The decoding algorithms typically used on multi-user channels are based on interference cancellation, and good performance with these approaches requires to be able to decode one of the user's message with reasonable fidelity, which in turn is used to decode the message of the other user, see for instance [17]. When the rates are not comparably low, to be able to achieve this low fidelity, one utilizes long block lengths [18] and/or unequal power allocation between users [17]. Indeed, in Section IV, we illustrate the importance of the long block length requirement: using the procedure based on soft interference cancellation in [13], [14] for decoding of short length rate 1/2 LDPC codes under equal power regime results in poor performance although this method provides very good results for long block lengths [13], [14].

While addressing these concerns regarding design and decoding of LDPC codes in short length scheme remains an interesting challenge, motivated by various factors, including the availability of ML decoding, here we have proposed a design methodology based on convolutional (trellis-based) codes. We illustrate the performance of the codes found by this proposed method in Section IV.

\section{SPecific Examples And Their Performance}

We now report on the performance obtained by the joint design scheme described in Section III. Decoding for both users is done jointly by the Viterbi algorithm, which provides an ML decoding solution for both users. In our examples, we also compare the performance of the proposed codes with some available LDPC codes. Since it is not possible to perform ML or MAP decoding for LDPC codes, and the channel is a MAC, we resort to the procedure in [13], [14] performing successive interference cancellation.

We define the signal-to-noise ratio as SNR $=\frac{\left|\alpha_{1}^{2}\right|}{N_{0} / 2}=\frac{\left|\alpha_{2}^{2}\right|}{N_{0} / 2}$ where $\alpha_{2}=\alpha_{1}$, unless otherwise stated. Here $\left|c_{t}^{i}\right|=1$ with BPSK modulation. To represent the generator polynomials of the convolutional codes, we use octal notation. A code pair for MAC channel is represented by putting the octal representations together, for instance, rate $1 / 2$ codes, for which the first user's code is $\left(o_{1}^{u_{1}}, o_{2}^{u_{1}}\right)$, is denoted by $\left(o_{1}^{u_{1}}, o_{2}^{u_{1}}\right) /\left(o_{1}^{u_{2}}, o_{2}^{u_{2}}\right)$.

We present the performance of the designed rate $1 / 2$ codes with constraint length 2 in Fig. 1 for $n=96$. We note that due to trellis termination, the code rate is $\approx 0.4792$. We observe that the order of magnitude of this frame length is consistent with the applications that motivated this study; such as voice communications with tight latency requirements or access channels that is used for call set-up in mobile environments. This particular choice is made due to availability of the off-the-shelf LDPC codes with this frame length.

Depending on the SNR regime of operation, two different code pairs are found by our proposed code search methodology: $\mathcal{C}_{1}=(6,3) /(5,5)$ (for high SNR), $\mathcal{C}_{2}=(6,7) /(7,5)$ (for low $\mathrm{SNR}$ ). To compare the performance of the proposed codes with the available convolutional codes designed for P2P channels, we consider the following "interleaved" scheme: one user encodes by using the given code, and for the second user, another assignment of the generator matrices to the output bits are used. (The generators are fixed, while the index of the output bit they generate changes.) In our example, we use the $(5,7)$ code which 


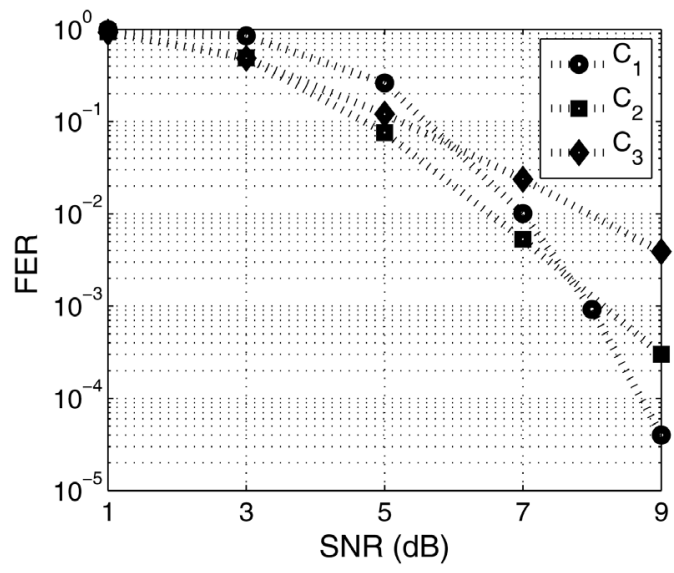

Fig. 1. Performance comparison of proposed codes, $R=1 / 2 . \mathcal{C}_{1}$ and $\mathcal{C}_{2}$ are designed codes, $\mathcal{C}_{3}$ is the code derived from optimal free distance rate $1 / 2$ code with constraint length 2 .

is the code with the largest minimum distance with constraint length 2. Hence the resulting MAC code is $\mathcal{C}_{3}=(5,7) /(7,5)$. Decoding is again done jointly for both users by the Viterbi algorithm. We observe that at relatively low SNRs, $\mathcal{C}_{3}$ performs very close to one of the designed codes and performs better than the other one. (We also note that the $\mathcal{C}_{3}$ appears as the second best code for low SNR after $\mathcal{C}_{2}$ in our code search.) On the other hand, for high SNR values, both of the jointly designed codes show superior performance compared to $\mathcal{C}_{3}$. We observe that to obtain a target FER value lower than 0.001 at $9 \mathrm{~dB}$, one needs to use one of the jointly designed codes.

The dependence of the relative performance of these codes on SNR may be interpreted in terms of the relative level of the interference from the other user and the noise. At relatively low SNRs, the dominant performance limiting factor is noise (rather than the other user's signal), hence the main element that determines a code's performance is its noise suppression capabilities. Hence a code designed for a P2P channel can exhibit good performance, as $\mathcal{C}_{3}$ does. However, as SNR becomes higher, the dominant element of interference becomes the other user's transmission. In this case, it is not optimal to treat the interference of the other user as noise, hence it becomes important that the codes jointly form a strong pair.

We have also compared the performance of the designed codes with some available short length LDPC codes. Codes for both users are chosen from LDPC codes with rate 0.5 from [19] with block length $n=96$. The code of one user is fixed as 96.3.963, and 5 different pairs are formed by choosing the code for the other user from $\{96.33 .964,96.3 .965,96.33 .966,96.3 .967,96.33 .968\}$. Due to their poor performance, error values for these LDPC codes are not shown in Fig. 1: at an SNR of 9 dB, FER values for all the above LDPC code pairs are observed to be larger than 0.1, whereas Fig. 1 shows a FER value in the order of $\approx 4 \times 10^{-5}$ for jointly designed convolutional codes. This poor performance of LDPC codes is consistent with the existence of many factors that may degrade their performance, including lack of joint design, lack of known joint ML decoding procedure, and the possible poor performance of the belief propagation based decoding scheme due to the presence of cycles in the short block length scheme.

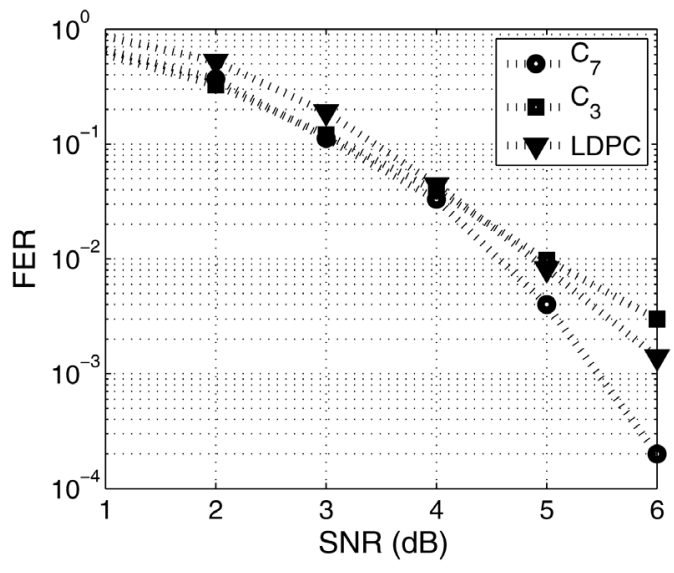

Fig. 2. Performance comparison of proposed codes, $R=1 / 2, \alpha_{1}=\sqrt{2} \alpha_{2}$. $\mathcal{C}_{7}$ is the designed code, $\mathcal{C}_{3}$ is the code derived from optimal free distance rate $1 / 2$ code with constraint length 2 , LDPC code from [19].

We now consider a scenario where the users have unequal power. Let $\alpha_{1}=\sqrt{2} \alpha_{2}$. The code search process results in the following code: $\mathcal{C}_{7}=(2,7) /(7,5)$. FER values versus the 2 nd user's SNR are presented in Fig. 2 along with the performance of $\mathcal{C}_{3}$, and the performance of the 3 rd LDPC code pair from the list above (which has the best performance at $6 \mathrm{~dB}$ while the performance of the other pairs are also close). While the LDPC code performance is substantially better than the case where the users have equal power (in terms of how close its performance is to the proposed convolutional code), the designed convolutional code pair still shows superior error performance. The fact that the performance gap between the proposed code $\left(\mathcal{C}_{7}\right)$ and the codes derived from P2P codes $\left(\mathcal{C}_{3}\right.$ and LDPC code) is smaller is again consistent with the expected effect of the dominant interference factor in the system. When one user's power level is low compared to the other, the interference caused by the low power user to the user with higher power level becomes relatively weak in comparison with the channel noise. Hence it becomes possible to decode the message of the high power user with a strong P2P code. This decoded message in turn helps to decode the message of the low power user, for instance, through the soft interference cancellation in the joint (belief propagation) decoding algorithm in the case of LDPC codes. We note that by using time-sharing between different rate point pairs, it may be possible to improve the performance of LDPC codes in equal power scenario through a similar effect. Here rate pairs where one of the users has a relatively low rate will be utilized to assist soft interference cancellation. However, the need for optimization of parameters such as rate pair choices together with the limited availability of short length LDPC codes with varying rates makes pursing this approach more challenging.

\section{CONCLUSIONS}

Trellis-based codes are designed for the two-user Gaussian MAC in the short block length regime. Our results show that the proposed codes achieve superior performance compared to the available codes designed for $\mathrm{P} 2 \mathrm{P}$ channels especially in the high SNR regime in equal power scenarios. 


\section{REFERENCES}

[1] L. Wei and H. Qi, "Near-optimal limited-search detection on ISI/CDMA channels and decoding of long convolutional codes," IEEE Trans. Inf. Theory, vol. 46, pp. 1459-1482, Jul. 2000.

[2] C. Shannon, "Probability of error for optimal codes in a Gaussian channel," Bell Syst. Tech. J., vol. 38, pp. 611-656, May 1959.

[3] Y. Polyanskiy, H. Poor, and S. Verdu, "Channel coding rate in the finite blocklength regime," IEEE Trans. Inf. Theory, vol. 56, pp. 2307-2359, May 2010.

[4] A. Stefanov and T. M. Duman, "Performance bounds for space-time trellis codes," IEEE Trans. Inf. Theory, vol. 49, no. 9, pp. 2134-2140, 2003.

[5] C. Ling, K. Li, and A. Kot, "Performance of space-time codes: Gallager bounds and weight enumeration," IEEE Trans. Inf. Theory, vol. 54, no. 8, pp. 3592-3610, 2008.

[6] V. Tarokh, N. Seshadri, and A. Calderbank, "Space-time codes for high data rate wireless communication: Performance criterion and code construction," IEEE Trans. Inf. Theory, vol. 44, no. 2, pp. 744-765, 1998.

[7] R. Blum, "Some analytical tools for the design of space-time convolutional codes," IEEE Trans. Commun., vol. 50, no. 10, pp. 1593-1599, 2002.

[8] Y. Li and B. Vucetic, "Optimization of space-time block codes based on multidimensional super-set partitioning," IEEE Signal Process. Lett., vol. 12, pp. 317-320, Apr. 2005.

[9] H. Sadjadpour, K. Kim, H. Wang, R. Blum, and Y. H. Lee, "Application of randomization techniques to space-time convolutional codes," IEEE Trans. Signal Process., vol. 54, pp. 4484-4489, Nov. 2006.
[10] V. Tralli, A. Conti, and M. Chiani, "Pragmatic space-time trellis codes: GTF-based design for block fading channels," IEEE Trans. Signal Process., vol. 59, pp. 2809-2823, Jun. 2011.

[11] S. Verdu, "Maximum likelihood sequence detection for intersymbol interference channels: A new upper bound on error probability," IEEE Trans. Inf. Theory, vol. 33, pp. 62-68, Jan. 1987.

[12] W. E. Ryan and S. Lin, Channel Codes: Classical and Modern. Cambridge, U.K.: Cambridge University Press, 2009.

[13] A. Roumy and D. Declercq, "Characterization and optimization of LDPC codes for the 2-user Gaussian multiple access channel," in EURASIP J. Wireless Commun. Netw., 2007.

[14] P. Berlin and D. Tuninetti, "LDPC codes for fading Gaussian broadcast channels," IEEE Trans. Inf. Theory, vol. 51, no. 6, pp. 2173-2182, 2005.

[15] A. Ramamoorthy and R. Wesel, "Construction of short block length irregular low-density parity-check codes," in IEEE Int. Conf. Communications, Jun. 2004, vol. 1, pp. 410-414.

[16] L. Wei, "Several properties of short LDPC codes," IEEE Trans. Commun., vol. 52, pp. 721-727, May 2004.

[17] N. Chayat and S. Shamai, "Iterative soft onion peeling for multi-access and broadcast channels," in IEEE Int. Symp. Personal, Indoor and Mobile Radio Communications, Sep. 1998, vol. 3, pp. 1385-1390.

[18] J. Boutros and G. Caire, "Iterative multiuser joint decoding: Unified framework and asymptotic analysis," IEEE Trans. Inf. Theory, vol. 48, pp. 1772-1793, Jul. 2002

[19] D. J. MacKay, "Encyclopedia of sparse graph codes," [Online]. Available: http://www.inference.phy.cam.ac.uk/mackay/codes/data.html 